\title{
The Differential Effects Fallacy in the Study of Self-compassion: Misunderstanding the Nature of Bipolar Continuums
}

\author{
Kristin D. Neff ${ }^{1}$ D \\ Accepted: 17 January 2022 / Published online: 31 January 2022 \\ (c) The Author(s), under exclusive licence to Springer Science+Business Media, LLC, part of Springer Nature 2022
}

\begin{abstract}
This brief commentary highlights the faulty reasoning underlying claims that selfcompassion should not be conceptualized or measured as a unitary construct because compassionate self-responding (CS) and uncompassionate self-responding (UCS) are differential predictors of psychological outcomes. The Self-Compassion Scale (SCS) is a well-validated measure that has been used in thousands of studies on selfcompassion. Self-compassion forms a bipolar continuum ranging from UCS (selfjudgment, isolation, and over-identification) to CS (self-kindness, common humanity, and mindfulness), so that higher SCS scores represent increased CS and reduced UCS. Several scholars have argued that because CS and UCS display different strengths of association with various psychological outcomes, that CS and UCS should be examined separately and a total SCS score is invalid. This conclusion is assumed to be self-evident. However, there is no known logical, scientific or psychometric principle that supports the view that opposite ends of a bipolar continuum must have the same strength of association with outcomes in order to operate as a unitary construct. In fact, it is common for opposite ends of a continuum to be differential predictors of outcomes (e.g., coldness is a stronger predictor of frostbite than warmth).Unfortunately, this unexamined assumption has spread widely in the field of selfcompassion research, setting the field back and muddying the waters for researchers. This commentary sheds light on this false premise in the hope of clearing up confusion.
\end{abstract}

Keywords Self-Compassion; Self-Warmth; Self-Coldness; Self-Compassion Scale: BipolarContinuum

The field of self-compassion research is exploding, with over 4000 journal articles and dissertations focused on the topic (based on a Google Scholar search of entries with "self-compassion" in the title, December 2021). The majority of research is conducted using the Self-Compassion Scale (SCS; Neff, 2003a, a multidimensional measure that includes three subscales representing compassionate selfresponding (CS) — self-kindness, common humanity, and mindfulness-and three subscales representing uncompassionate self-responding (UCS) — self-judgment, isolation, and over-identification. Self-compassion is conceptualized as a bipolar continuum ranging from UCS to CS, and UCS items are reverse-coded so that higher levels of self-compassion represent reduced UCS. Note that UCS dimensions do not merely represent the lack of CS. Instead, the UCS and CS dimensions are qualitatively distinct and are thought to

Kristin D. Neff

kneff@austin.utexas.edu

1 Educational Psychology, The University of Texas at Austin, 1912 Speedway, Suite 504, Austin, TX 78712-1289, USA have different causes, correlates, and manifestations (Neff, 2016).

There has been controversy in the field of self-compassion research over whether CS and UCS operate as a unitary construct or as two independent, orthogonal constructs. This controversy partly stems from misunderstanding the nature of continuums. A bipolar continuum is composed of two qualitatively distinct opposites that range from -1 to 1 (Tay \& Jebb, 2018). Heat and cold are a prototypical bipolar continuum. One feature of a bipolar continuum is that observations can fall in a range on either side of the zero pointvery hot, a little warm, somewhat cold, not at all cold, etc. Another feature is that one can be low in one quality yet low, medium, or high in the opposite quality (Tay \& Drasgow, 2012). For instance, lukewarm water is low in cold and low in heat, while hot water is low in cold and high in heat. This means that there is not always a high correlation between observations falling on each side of the zero point when measured separately because variation on one side does not necessarily imply variation in the other. In contrast, unipolar continuums range from 0 to 1 and describe only one quality 
without a neutral zero point. For example, movement and stillness form a unipolar continuum. Stillness is the zero point and observations on the continuum measure variation in movement alone.

Some researchers have found that CS and UCS form separate factors in psychometric analyses of the SCS (Brenner et al., 2017; Costa et al., 2016; López et al., 2015) and concluded that they form two orthogonal unipolar continuums which should not be combined into a total self-compassion score. However, statistically testing the factor structure of bipolar continuums using conventional methods can be misleading, as they tend to split bipolar continuums in half (Tay \& Drasgow, 2012). Fortunately, sophisticated methods such as bifactor exploratory structural equation modeling (ESEM; Morin et al., 2016) appear better able to model the factor structure of bipolar continuums, especially when they are multidimensional. This method has been used to analyze similar bipolar constructs such as extrinsic/intrinsic motivation (Howard et al., 2018) or need frustration/satisfaction (Tóth-Király et al., 2019), and support has been found for the conclusion that these constructs operate as a single bipolar continuum rather than two separate unipolar continuums. Bifactor ESEM has been used to examine the SCS in over 20 samples (Neff et al., 2019; Tóth-Király \& Neff, 2021), with findings indicating that self-compassion is best explained by one general factor (representing a bipolar continuum) rather than two separate CS and UCS factors (representing two orthogonal unipolar continuums). These studies have also found that over $90 \%$ of the reliable variance in the SCS is explained by one general factor. I will not address psychometric issues in depth here as they have been discussed extensively elsewhere (Neff, 2019; Neff \& Tóth-Király, 2022). My main purpose here is to highlight a particular fallacy of reasoning that has been unquestioned by many in field: the differential effects fallacy.

Some scholars-most notably Muris and his colleagues (Muris, 2016; Muris et al., 2016; Muris et al., 2019; Muris \& Petrocchi, 2017) - have asserted that the well-established finding that UCS items predict psychopathology (e.g., depression, anxiety) more powerfully than CS items (Muris \& Petrocchi, 2017; Neff et al., 2018) necessarily means that CS and UCS form two independent unipolar continuums that should be examined separately. Moreover, it has been argued that because UCS items are the main drivers of the inverse association between total SCS scores and psychopathology, this "inflates" the link between self-compassion and psychopathology and UCS items should be dropped from the SCS (Muris \& Petrocchi, 2017). These conclusions are presumed to be so self-evident that Muris and Otgaar (2020) wrote a commentary in Mindfulness claiming that "there is irrefutable proof that the current conceptualization of self-compassion and the way this trait is currently assessed with the SCS are inappropriate" (p. 1479) and that research on self-compassion as a unitary construct is "conducted by human beings who do not always operate in a logical, rational way but rather are driven by personal interests, cognitive biases, and social influences" (p. 1476).

Although the evidence strongly suggests that UCS predicts psychopathology more powerfully than CS, this does not mean that self-compassion must be assessed as two separate constructs. There is no known logical, scientific, or psychometric principle that supports the claim that variation at opposite ends of a bipolar continuum must have the same strength of association with outcomes in order to be examined as a unitary construct. Theoretically, if the only difference between CS and UCS was reverse-coding and they were thought to represent a single unipolar continuum ranging from 0 to 1 , there could be some merit to this claim. But self-compassion is a bipolar continuum with qualitatively distinct endpoints, meaning that there is no logical reason why variation at each end of the continuum should evidence the same strength of association with outcomes. Unfortunately, the differential effects fallacy has muddied and confused the field of self-compassion research.

There have been dozens of studies that have used CS and UCS items as separate predictors of outcomes operating in accord with this fallacy. Typically, it is found that UCS and CS show different strengths of association with various constructs such as distress and well-being (Brenner et al, 2018), depression (López et al, 2018), insecure attachment (Brophy et al., 2020), help-seeking (Dschaaket al., 2021), post-traumatic growth (Wong \& Yeung, 2017), and life satisfaction (Charzyńska et al., 2020). Rather than simply interpreting these findings as illustrating the mechanisms of action of self-compassion (e.g., that variation at one end of the continuum more strongly impacts outcomes than variation at the other end), study authors typically conclude that CS and UCS must be examined separately rather than being combined into a total SCS score. Moreover, even though these studies have largely been based on cross-sectional data, researchers also commonly conclude that findings have important clinical implications and suggest that UCS and CS should be targeted separately in intervention.

As an example, Bicaker and Racine (2022) conducted a study examining the link between self-compassion and disordered eating behavior using separate UCS and CS scores. Unsurprisingly, they found that UCS explained more of the variance in disordered eating, as has been shown in other research (e.g., Neff et al., 2018). However, they interpreted their findings to mean that "results highlight the importance of examining compassionate and uncompassionate selfresponding separately in relation to eating disorder behaviors and of potentially targeting uncompassionate versus compassionate self-responding in eating disorder interventions" (p. 5). While they note that compassion-based interventions such as Compassion-Focused Therapy (CFT) are effective 
in treating eating disorders and have been shown to reduce UCS, they fail to mention that the way that compassionbased interventions such as CFT reduce UCS among eating disordered populations is by increasing CS (Steindl et al., 2017). It does not make sense to target UCS and CS separately because they change in tandem: As CS increases, UCS decreases.

The two main self-compassion-based interventions that have been developed-CFT (Gilbert, 2009) and mindful self-compassion (MSC; Neff \& Germer, 2013) - both explicitly use CS to reduce UCS. According to Gilbert (2009), the goal of CFT is "to stimulate feelings of safeness, warmth and connectedness [CS]" in order to counteract the UCS entailed by over-arousal of the threat system, or as Gilbert puts it, to "replace self-criticism with self-kindness." Similarly, in MSC, self-compassion practices such as the self-compassion break are designed to reduce UCS by increasing feelings of kindness, connectedness and mindfulness (CS) in response to experiences of suffering (Germer $\&$ Neff, 2019). If CS and UCS were truly independent and orthogonal constructs, CFT and MSC would not work nearly as effectively as they do (Germer \& Neff, 2019; SommersSpijkerman et al., 2018).

Among those who argue that CS and UCS are orthogonal and unrelated, CS is sometimes referred to as self-warmth and UCS as self-coldness (Brenner et al., 2018; Brophy et al., 2020; Costa et al., 2015; López et al., 2015). I argued (Neff, 2016) that the terms CS and UCS were more appropriate because warmth and coldness primarily refer to kindness and self-judgment and overlook the other self-compassion components. For my purposes here, however, these terms are useful for illustrating the differential effects fallacy as applied to the bipolar continuum of physical warmth and coldness. As a thought experiment, imagine that you were interested in examining the impact of temperature on health among people living in different climates. If you did not have access to thermometer readings, you might conduct a study with a subjective measure of temperature using items like "I feel cold," "I get chilly," "I feel warm," and "I get hot" using a response scale of almost never to almost always. To calculate a total subjective temperature score, you would reverse code the coldness items so that increasing temperature represented more warmth and less coldness. Let us say you also gathered data on how often people experienced different outcomes like frostbite or heatstroke. If you were to conduct such a study, you would find that higher total temperature scores evidenced a negative association with frostbite and a positive association with heatstroke. If you calculated separate warmth and coldness scores, you would find that coldness had a stronger association with frostbite and that warmth had a stronger association with heatstroke. It is useful to know that coldness primarily explains frostbite and warmth primarily explains heat stroke. This information is relevant to understanding the mechanisms of action of temperature. It would be strange, however, to argue that these findings mean that coldness and warmth do not form a unitary construct of temperature, or that the use of a total temperature score was invalid, or that inclusion of coldness items inflates the link between temperature and frostbite. It would also be odd to draw the conclusion that coldness and warmth should be targeted separately in an intervention to treat frostbite because the way to make things less cold is to warm them up.

If the logic that two ends of a continuum cannot differentially predict outcomes is false for a simple unidimensional construct like temperature, it is most certainly false for a complex, multidimensional construct like self-compassion. For instance, given that self-judgment, feelings of isolation, and over-identification directly contribute to negative outcomes like depression (Luyten et al., 2007; Nolen-Hoeksema, 2000; Santini et al., 2020), it should be expected that variations in UCS items are stronger predictors of psychopathology than CS items. The first paper I wrote on selfcompassion (Neff, 2003b) posited that the main way selfcompassion works to reduce psychopathology is through decreased UCS. This in no way shape or form invalidates the use of a total SCS score or the view that self-compassion forms a bipolar continuum ranging from UCS to CS.

In addition to psychometric analyses, one way to identify whether a construct operates as a single bipolar continuum or as two orthogonal continuums is by establishing whether changes in responses on each dimension occur in a single direction or if they can occur in opposite directions (Tay \& Jebb, 2018). There is a large body of research on self-compassion interventions that show gains in trait self-compassion manifest as a movement along a continuum in a single direction: as individuals increase in CS, they decrease in UCS (Ferrari et al., 2019; Wilson et al., 2019). While most of this research has examined change in trait SCS scores, changes in state SCS scores have even more relevance to examining self-compassion as a construct because it measures responses to a single situation as opposed to different situations over time. Neff et al. (2021) examined change in state SCS scores after a self-compassion mood induction, which involved writing a paragraph expressing kindness, remembering common humanity and being mindful about a particular instance of suffering. Results indicated that selfkindness increased $10 \%$ and self-judgment decreased $10 \%$; common humanity increased $12 \%$ and isolation decreased $11 \%$; mindfulness increased $9 \%$ and over-identification decreased $8 \%$. Similarly, Miyagawa et al. (2022) examined change in state self-compassion after a mood induction in a Japanese sample and found that self-kindness increased $30 \%$ and self-judgment decreased $28 \%$; common humanity increased 24\% and isolation decreased 31\%; mindfulness increased $24 \%$ and over-identification decreased $29 \%$. If CS 
and UCS were orthogonal, it would mean that some participants would simultaneously increase their endorsement of CS items such as "I' $m$ giving myself the tenderness and caring I need," "I'm remembering that there are lots of others in the world feeling like I am," or "I'm taking a balanced view of this painful situation," and UCS items such as "I'm being pretty tough on myself," "I feel like I'm struggling more than others right now," or "I' $m$ blowing this painful incident out of proportion." Such a pattern of responses would be nonsensical because CS and UCS are polar opposites.

Of course, it is useful to know that variation at each end of the self-compassion continuum differentially predicts outcomes. For instance, findings may mean that it is less useful to focus on increasing CS when treating outcomes such as anxiety and depression after a certain reduction in UCS has been reached. On the other hand, given that those with high levels of CS are likely to be more protected against psychopathology, it may be that higher levels of self-compassion help to prevent relapse. A big gap in the field involves establishing cutoff values on the SCS that indicate risk for or protection against psychopathology, and the question of whether this is best done with a total score, the six subscale scores, or even a separate CS and USC score is yet to be answered.

The differential effects fallacy is potentially harmful to the field of self-compassion research for several reasons. First, the use of separate UCS and CS scores instead of one SCS score may sometimes lead to confusing and convoluted findings that obscure the fact that UCS and CS operate in tandem and form a single bipolar continuum. Unless one is interested in self-compassion's mechanisms of action, use of a total SCS score more comprehensively represents how taking a self-compassionate approach to suffering (i.e., increasing CS and reducing UCS) may affect outcomes such as life satisfaction, depression, and resilience. Another problem is that it greatly reduces the variance that is measured in self-compassion if scholars use each half of the scale separately, or adopt the recommendation to drop the UCS items from the SCS scale altogether. It is good psychometric practice to include items that describe the full range of possible response options along a continuum in order to maximize the variance observed (Tay \& Jebb, 2018). Studies that only use CS items to examine the association between self-compassion and psychopathology under the mistaken assumption that self-compassion does not involve reduced UCS, for instance, might assume that self-compassion is not that relevant to coping with distress. Results would be misleading because effect sizes found for self-compassion would appear smaller than they really are.

Such misinterpretations could lead to what may be the biggest danger of the differential effects fallacy: it may have a chilling effect (no pun intended) on those wanting to use self-compassion as an intervention. Researchers conducting cross-sectional studies who find that the link between selfcompassion and psychopathology is primarily explained by UCS might assume that self-compassion interventions are ineffective for outcomes like anxiety and depression, despite the large body of intervention research which shows the contrary (Ferrari et al., 2019; Wilson et al., 2019). The advice given by some researchers to target interventions toward either lessening UCS or increasing CS could also be confusing for the field, given that compassion-based interventions such as CFT and MSC decrease UCS and precisely by increasing CS! Why do scholars like me care so much about self-compassion? Because it works. People can practice self-compassion whenever suffering is present, meaning they give themselves support by being kind, connected, and mindful, and reduce their suffering by being less judgmental, feeling isolated, or over-identifying with the pain. It is my hope that the field can turn its attention toward issues that have actual implications for bettering lives (like establishing clinical cutoff scores), and spend less time conducting studies that simply reinforce the differential effects fallacy. The fact that CS and UCS differentially predict outcomes has no bearing on whether or not self-compassion forms a bipolar continuum or if it can be measured as a unitary construct.

\section{Declarations}

Conflict of Interest The author declares no competing interests.

Ethics Approval The manuscript does not involve human or animal participants.

\section{References}

Bicaker, E., \& Racine, S. E. (2022). Protection versus risk? The relative roles of compassionate and uncompassionate self-responding for eating disorder behaviors. Eating Behaviors, 44, 101592.

Brenner, R. E., Heath, P. J., Vogel, D. L., \& Credé, M. (2017). Two is more valid than one: Examining the factor structure of the Self-Compassion Scale (SCS). Journal of Counseling Psychology, 64(6), 696-707.

Brenner, R. E., Vogel, D. L., Lannin, D. G., Engel, K. E., Seidman, A. J., \& Heath, P. J. (2018). Do self-compassion and self-coldness distinctly relate to distress and well-being? A theoretical model of self-relating. Journal of Counseling Psychology, 65(3), 346-357.

Brophy, K., Brähler, E., Hinz, A., Schmidt, S., \& Körner, A. (2020). The role of self-compassion in the relationship between attachment, depression, and quality of life. Journal of Affective Disorders, 260, 45-52.

Charzyńska, E., Kocur, D., Działach, S., \& Brenner, R. E. (2020). Testing the indirect effect of type 1 diabetes on life datisfaction through selfcompassion and self-coldness. Mindfulness, 11(11), 2486-2493.

Costa, J., Marôco, J., Pinto-Gouveia, J., Ferreira, C., \& Castilho, P. (2016). Validation of the psychometric properties of the SelfCompassion Scale. Testing the factorial validity and factorial invariance of the measure among borderline personality disorder, 
anxiety disorder, eating disorder and general populations. Clinical Psychology \& Psychotherapy, 23, 460-468.

Dschaak, Z. A., Spiker, D. A., Berney, E. C., Miller, M. E., \& Hammer, J. H. (2021). Collegian help seeking: The role of self-compassion and self-coldness. Journal of Mental Health, 30(3), 284-291.

Ferrari, M., Hunt, C., Harrysunker, A., Abbott, M. J., Beath, A. P., \& Einstein, D. A. (2019). Self-compassion interventions and psychosocial outcomes: A meta-analysis of RCTs. Mindfulness, 10, 1455-1473.

Germer, C. K., \& Neff, K. D. (2019). Teaching the Mindful Self-Compassion program: A guide for professionals. Guilford Press.

Gilbert, P. (2009). Introducing compassion-focused therapy. Advances in Psychiatric Treatment, 15(3), 199-208.

Gilbert, P., McEwan, K., Matos, M., \& Rivis, A. (2011). Fears of compassion: Development of three self-report measures. Psychology and Psychotherapy, 84, 239-255.

Howard, J. L., Gagné, M., Morin, A. J. S., \& Forest, J. (2018). Using bifactor exploratory structural equation modeling to test for a continuum structure of motivation. Journal of Management, 44, 2638-2664.

López, A., Sanderman, R., Smink, A., Zhang, Y., van Sonderen, E., Ranchor, A., \& Schroevers, M. J. (2015). A reconsideration of the SelfCompassion Scale's total score: Self-compassion versus self-criticism. PloS One, 10(7). https://doi.org/10.1371/journal.pone.0132940

López, A., Sanderman, R., \& Schroevers, M. J. (2018). A close examination of the relationship between self-compassion and depressive symptoms. Mindfulness, 9(5), 1470-1478.

Luyten, P., Sabbe, B., Blatt, S. J., Meganck, S., Jansen, B., De Grave, C., ... \& Corveleyn, J. (2007). Dependency and self-criticism: Relationship with major depressive disorder, severity of depression, and clinical presentation. Depression and Anxiety, 24(8), 586-596.

Miyagawa, Y., Tóth-Király, I., Knox, M., Taniguchi, J. \& Niiya, Y. (2022). Development of the Japanese version of the State SelfCompassion Scale (SSCS-J). Frontiers in Psychology. doi: https:// doi.org/10.3389/fpsyg.2021.779318

Morin, A. J. S., Arens, A. K., \& Marsh, H. W. (2016). A bifactor exploratory structural equation modeling framework for the identification of distinct sources of construct-relevant psychometric multidimensionality. Structural Equation Modeling: A Multidisciplinary Journal, 23(1), 116-139.

Muris, P. (2016). A protective factor against mental health problems in youths? A critical note on the assessment of self-compassion. Journal of Child and Family Studies, 25, 1461-1465.

Muris, P., \& Otgaar, H. (2020). The process of science: A critical evaluation of more than 15 years of research on self-compassion with the Self-Compassion Scale. Mindfulness, 11(6), 1469-1482.

Muris, P., Otgaar, H., Meesters, C., Heutz, A., \& Van den Hombergh, M. (2019). Self-compassion and adolescents' positive and negative reactions to daily life problems. Journal of Child and Family Studies, 28, 1433-1444.

Muris, P., Otgaar, H., \& Petrocchi, N. (2016). Protection as the mirror image of psychopathology: Further critical notes on the selfcompassion scale. Mindfulness, 7(3), 787-790.

Muris, P., \& Petrocchi, N. (2017). Protection or vulnerability? A meta-analysis of the relations between the positive and negative components of self-compassion and psychopathology. Clinical Psychology \& Psychotherapy, 24(2), 373-383.

Neff, K. D. (2003a). Development and validation of a scale to measure self-compassion. Self and Identity, 2, 223-250.

Neff, K. D. (2003b). Self-compassion: An alternative conceptualization of a healthy attitude toward oneself. Self and Identity, 2, 85-102.

Neff, K. D. (2016). The Self-Compassion Scale is a valid and theoretically coherent measure of self-compassion. Mindfulness, 7(1), 264-274.

Neff, K. D. (2019). Setting the record straight about the Self-Compassion Scale. Mindfulness, 10, 200-202.

Neff, K., \& Germer, C. (2013). A pilot study and randomized controlled trial of the mindful self-compassion program. Journal of Clinical Psychology, 69(1), 28-44.
Neff, K. D., Long, P., Knox, M., Davidson, O., Kuchar, A., Costigan, A., Williamson, Z., Rohleder, N., Tóth-Király, I., \& Breines, J. (2018). The forest and the trees: Examining the association of self-compassion and its positive and negative components with psychological functioning. Self and Identity, 17(6), 627-645.

Neff, K. D. \& Tóth-Király (2022). The Self-Compassion Scale. In O. N. Medvedev, C. U. Krägeloh, R. J. Siegert and N. N. Singh (Eds.) Handbook of assessment in mindfulness research. New York: Springer.

Neff, K. D., Tóth-Király, I., Knox, M. C., Kuchar, A., \& Davidson, O. (2021). The development and validation of the State Self-Compassion Scale (Long-and Short Form). Mindfulness, 12(1), 121-140.

Neff, K. D., Tóth-Király, I., Yarnell, L., Arimitsu, K., Castilho, P., Ghorbani, N., Guo, H. X., Hirsch, J., Hupfeld, J., Hutz, C., Kotsou, I., Lee, W. K., Montero-Marin, J., Sirois, F., de Souza, L., Svendsen, J., Wilkinson, R., \& Mantios, M. (2019). Examining the factor structure of the Self-Compassion Scale using exploratory SEM bifactor analysis in 20 diverse samples: Support for use of a total score and six subscale scores. Psychological Assessment, 31(1), 27-45.

Nolen-Hoeksema, S. (2000). The role of rumination in depressive disorders and mixed anxiety/depressive symptoms. Journal of Abnormal Psychology, 109(3), 504.

Santini, Z. I., Jose, P. E., Cornwell, E. Y., Koyanagi, A., Nielsen, L., Hinrichsen, C., Meilstrup, C., Madsen, K. R., \& Koushede, V. (2020). Social disconnectedness, perceived isolation, and symptoms of depression and anxiety among older Americans (NSHAP): A longitudinal mediation analysis. The Lancet Public Health, 5(1), e62-e70.

Sommers-Spijkerman, M., Trompetter, H., Schreurs, K., \& Bohlmeijer, E. (2018). Pathways to improving mental health in compassionfocused therapy: Self-reassurance, self-criticism and affect as mediators of change. Frontiers in Psychology, 9, 2442.

Steindl, S. R., Buchanan, K., Goss, K., \& Allan, S. (2017). Compassion focused therapy for eating disorders: A qualitative review and recommendations for further applications. Clinical Psychologist, 21(2), 62-73.

Tay, L., \& Drasgow, F. (2012). Theoretical and statistical issues in the assessment of construct dimensionality: Accounting for the item response process. Organizational Research Methods, 15, 363-384.

Tay, L., \& Jebb, A. T. (2018). Establishing construct continua in construct validation: The process of continuum specification. Advances in Methods and Practices in Psychological Science, 1(3), 375-388.

Tay, L., \& Kuykendall, L. (2017). Why self-reports of happiness and sadness may not necessarily contradict bipolarity: A psychometric review and proposal. Emotion Review, 9, 146-154.

Tóth-Király, I., Bőthe, B., Orosz, G., \& Rigó, A. (2019). A new look on the representation and criterion validity of need fulfillment: Application of the bifactor exploratory structural equation modeling framework. Journal of Happiness Studies, 20, 1609-1626.

Tóth-Király, I., \& Neff, K. D. (2021). Is self-compassion universal? Support for the measurement invariance of the Self-Compassion Scale across populations. Assessment, 28(1), 169-185.

Wilson, A. C., Mackintosh, K., Power, K., \& Chan, S. W. (2019). Effectiveness of self-compassion related therapies: A systematic review and meta-analysis. Mindfulness, 10(6), 979-995.

Wong, C. C. Y., \& Yeung, N. C. (2017). Self-compassion and posttraumatic growth: Cognitive processes as mediators. Mindfulness, 8(4), 1078-1087.

Publisher's Note Springer Nature remains neutral with regard to jurisdictional claims in published maps and institutional affiliations. 